\section{Discourse Semantics}

P A M Seuren, Max Planck Institute for

Psycholinguistics, Nijmegen, the Netherlands

(c) 2006 Elsevier Ltd. All rights reserved.

\section{Introduction}

Discourse Semantics (DSx) holds that the meaning of a sentence $A$ makes $A$ usable only in a certain class of contexts called discourse domains or Ds, built up on the basis of preceding utterances and available situational and world knowledge. The production of a meaningful utterance by a speaker $S$ starts with S's intent, in terms of a given $\mathrm{D}$, to make public a proposition - that is, a mental structure in which $\mathrm{S}$ assigns a property to one or more objects - under some form of socially binding commitment or appeal, the intended force or speech act type. The intent is fed into the language machinery, which categorizes and structures the cognitive elements in such a way that a sentence is formed, then realized as an utterance. Utterance comprehension involves the reconstruction of S's intent (force plus proposition) by a listener L. The intent conveyed by each new utterance is incremented to $\mathrm{D}$, which thus changes with each new increment. The first to propose mechanisms of this kind were Seuren (1972, 1975), Isard (1975), and Gazdar (1979). Since no theory of the force element in Ds is available, the present article is restricted to the incrementation of propositions only, implicitly assuming an overarching assertive (truth-vouching) force.

The incrementation of an uttered sentence $A$, or $\mathrm{i}(\mathrm{A})$, is achieved in terms of addresses mentally representing the objects or sets of objects mentioned in $A$. These objects are of widely divergent kinds, including (sets of (sets of)) individuals, substances, facts, and all kinds of abstractions or reifications constructed by the mind as 'objects.' A special kind of 'object' is represented by subdomains. A subdomain represents a thought-up scenery of its own, to which a property has been assigned in the proposition at hand. In John believes that the earth is flat or It is unlikely that the earth is flat, the information conveyed by that the earth is flat is stored in a subdomain representing what John believes or what is unlikely. Besides addresses, a D may contain instructions, which constrain its further development. Negation of a sentence $A$, for example, results in an instruction to block $\mathrm{i}(\mathrm{A})$ in $\mathrm{D}$. In this perspective, the linguistic meaning of a sentence $A$ is the contribution potential of $i(A)$ to any given $D$ in virtue of A's linguistically defined properties. More formally, the linguistic meaning of a sentence $A$ is a function from given $D$ s to incremented Ds.

The fact that interpretation is co-determined by a given $\mathrm{D}$ is reflected mainly by three kinds of phenomena (a) identification across domains and subdomains, (b) anaphora phenomena and (c) presuppositions.

Identification across (sub)domains is illustrated by a sentence like The girl with brown eyes has blue eyes, which seems inconsistent and hence uninterpretable. Yet it makes perfect sense if interpreted as 'the girl represented in the picture with brown eyes in reality has blue eyes,' or as 'the girl who in reality has brown eyes is represented in the picture as having blue eyes.' Fauconnier (1985) posits 'mental spaces,' autonomous but interconnected cognitive domains of interpretation whose elements are open to denotation by definite NPs under certain conditions.

Anaphora occurs when an anaphoric expression, usually a pronoun, takes over the (constant or variable) reference function of another expression, its antecedent, whose reference function is independently grounded. In DSx, an anaphoric expression selects the correct D-address via its antecedent.

Presupposition, neglected in other semantic theories, is central to DSx. A presupposition $A$ of a sentence $B$ is a semantic property of $B$ restricting its usability to those contexts to which $A$ has already been incremented or which admit the incrementation of $A$ without inconsistency or incompatibility with available knowledge.

A sentence must be anchored in its context or D to be interpretable. Moreover, when used seriously (not in play or fiction), it requires a force field in which the social position-taking is valid, and the proposition expressed must be intentionally focused or keyed to a verification domain $\mathrm{V}$ in the world. Anchoring accounts for interpretation; the force field accounts for the social position-taking; keying accounts for truth or falsity. The building up of any D, whether serious or in play or fiction, is subject to the condition that it must be possible for $\mathrm{D}$ to be socially valid and true. This makes social liability conditions and truth conditions directly relevant to the analysis of meaning.

The semantic analysis (SA) of the sentence to be incremented is the input to the incrementation procedure. The SA is a level of semantic representation consisting of a speech-act operator expressing the social position-taking and a semantically regular linguistic expression of the proposition, in terms of a variety of modern predicate calculus. Surface structures (SSs) too often conceal or distort their meanings 
(see Seuren, 1985: 61-110), which makes them unfit for direct semantic interpretation. A grammar $G$ is required relating SSs to their corresponding SAs and vice versa.

DSx thus posits two intermediate stages between SSs and whatever they be about in any V: the linguistic object $S A$ and the mental proposition anchored in a given $\mathrm{D}$. This $\mathrm{D}$ has open access to a knowledge base $(\mathrm{KB})$ of encyclopedic and situational knowledge. A set of incrementation rules (IC) relates SAs to Ds and vice versa. The overall structure of the theory is presented in Figure 1.

DSx is divided into two main sections, incrementation and subdomain structures. The former deals with the incrementation procedure of linguistic clauses to the appropriate (sub)domains, the latter with the embedding of subdomains.

\section{Incrementation}

The serial incrementation of sentences in a D is subject to semantic conditions, the most basic being the sequentiality condition (SC). SC does not apply to actual texts, which are hardly ever fully sequential (they would be unbearably verbose if they were), but to Ds. SC consists of three subconditions, presuppositional precedence, consistency, and informativity. The first requires that presuppositions are incremented before their carrier sentence. The second requires that a $\mathrm{D}$, at any stage, must have the possibility of being true and thus must be logically and semantically consistent. The third requires every new increment to be informative - that is, the set of situations in which D is true gets further restricted with each new increment (see Presupposition). Thus, sequences of the form 'A and possibly A', or 'A and (A or B)' are, though logically coherent (the first conjunct entails the second), discoursewise unacceptable. Besides SC there are other structuring principles for texts and Ds not discussed here. Foremost among these is the principle of topic-comment modulation, based on the notion that each new sentence in a discourse is meant as an answer to an often implicit question. The emphasis in this section is on definite reference to individuals, quantification, and plurality.

Consider, as a possible starting point of $\mathrm{D}$, the deceptively simple sentence (1a), with its (matrix-S) SA (1b), represented as a linguistic tree structure in (1c), and resulting in the $\mathrm{D}$-address (1d):

(1a) There was a cat.

(1b) $\mathrm{an}_{\mathrm{x}}[\mathrm{be}(\mathrm{x}), \operatorname{cat}(\mathrm{x})]$

(1c)

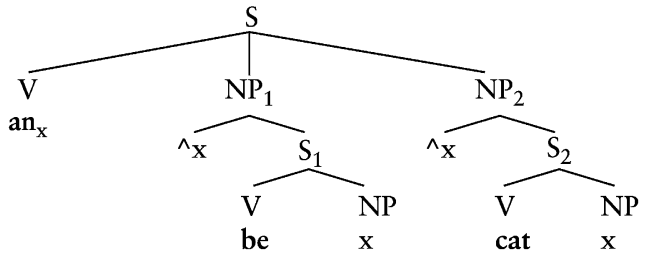

(1d) d-1 [a $\mid \operatorname{Cat}(a)]$

In (1b and 1c), an is the existential quantifier (aka $\exists$ ), treated as a binary higher-order predicate over pairs of sets, with the subject term $b e(x)$ and the object term $\operatorname{cat}(x)$. The index $x$ in $a n_{x}$ binds the variables $x$ in $b e(x)$ and $C a t(x)$. An requires for truth that there be at least one element common to the sets denoted by the two terms.

In standard logic, the existential quantifier posits actual existence of the common element. But that will not do for language, given sentences like (2a), which do not entail that the cat in question actually existed:

MIND

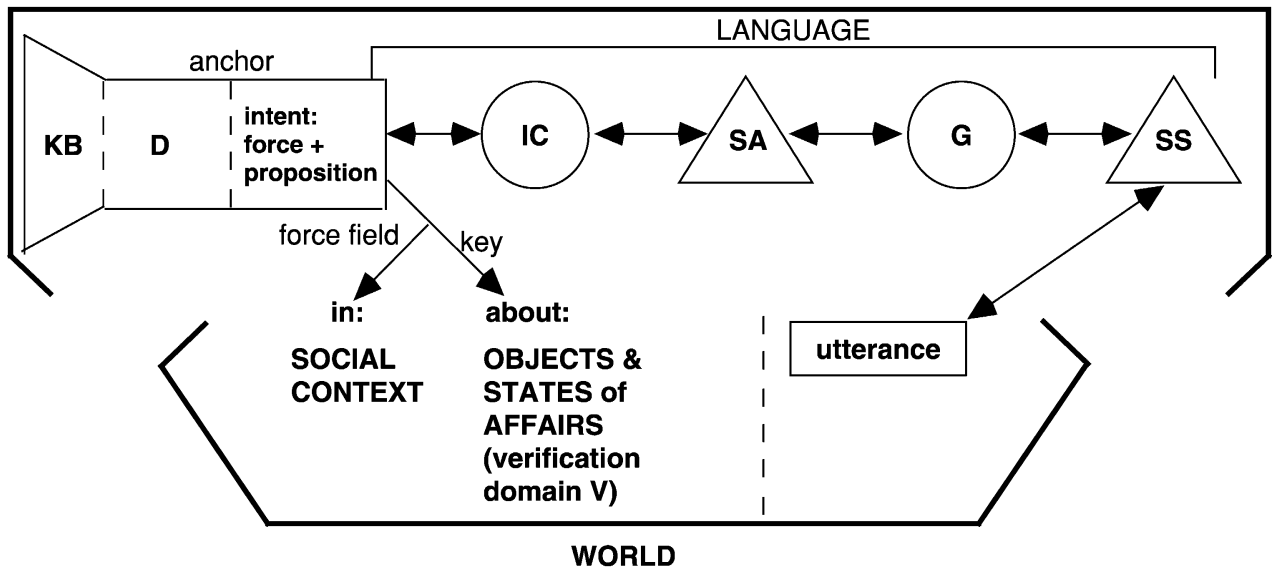

Figure 1 Overall structure of the theory. 
(2a) A cat was worshipped there.

(2b) $\operatorname{an}_{\mathrm{x}}[$ be worshipped(x), \#cat $(\mathrm{x})]$

(2c) d-2 [a $\mid$ \#Cat(a), Be worshipped(a)]

(3a) A child laughed.

(3b) $\operatorname{an}_{x}[\operatorname{laugh}(x), \operatorname{child}(x)]$

(3c) d-3 [a $\mid \operatorname{Child(a),\operatorname {Laugh}(a)]}$

For the semantics of language, it is stipulated that when one term of the existential quantifier has an intensional predicate denoting a cognitive process and capable of yielding truth also for thought-up entities (Frege's thought predicates), while the other term is extensional, yielding truth only for actually existing objects, the extensional term is intensionalized, so that it applies to thought-up entities as well. Since in (3b) both terms are extensional by nature, as only really existing individuals can truthfully be said to laugh or to be a child, (3a) is rendered as (3b). But in $(2 \mathrm{a})$ be worshipped is a cognitive intensional predicate as it may yield truth also for fictitious objects. Therefore, $\operatorname{cat}(x)$ is intensionalized to \#cat $(x)$, where \# indicates that the set denoted by \#cat $(x)$ may also contain virtual (thought-up) cats. This allows true existential quantification over virtual objects (see Virtual Objects).

$B e(x)$ in (1) is taken to be intensional by nature (and thus not marked by \#), denoting the axiomatically given nonnull set of actual or virtual objects that 'are there.' But since $b e(x)$ is not a thought predicate, it does not intensionalize the other term under an but is itself extensionalized when the other term is extensional. This ensures an entailment of actual existence for extensional terms, but also the absence of such an entailment for intensional terms, as in:

(4) There was an imaginary cat.

The SA-structure (1c) is the tree-structure counterpart of $(1 \mathrm{~b}) . \mathrm{NP}_{1}$ is the subject term, $\mathrm{NP}_{2}$ the object term, and ${ }^{\wedge}$ is a set-denoting operator: 'the set of things $x$ such that ...' The grammatical process transforming (1c) into (1a) is not at issue here. (Roughly, $\mathrm{NP}_{2}$ is incorporated into $\mathrm{v}[\mathrm{an}]$, forming the complex predicate $_{\mathrm{V}}\left[\mathrm{an}^{-}{ }^{\wedge} \mathrm{x}[\operatorname{cat}(\mathrm{x})]\right]$, which is then lowered into the position of the subject term $x$ of $b e$. For details, see Seuren, [1996: 300-309].)

The present concern is the incrementation procedure IC turning (1c) into the D-address (1d). IC scans the SA-predicate first. $A n$ is an instruction to create a new singular address (a first-order address over individuals). An address label $\mathbf{d}-\mathbf{1}$ is set up, identifying the address for later reference. The contents of the address is given between large square brackets. Here ' $a$ ' is the address head, representing an and binding the variables. It stands for the first-order existential quantifier
- a function from sets of individuals to truth values, typed $((e, t), t)$. The object term is incremented first, giving $\mathbf{d}-\mathbf{1}[\mathbf{a} \mid \operatorname{Cat}(\mathrm{a})]$. Normally, the subject term is then added, but not in the case of $b e(x)$, it being axiomatically understood that there are (actual or virtual) things. Therefore, only $\mathrm{NP}_{2}$ is incremented after the upright bar indicating the scope of a. (One notes the structural - but not the semantic - analogy of d-1 with the Russellian formula $\exists \mathrm{x}[\operatorname{cat}(\mathrm{x})]$.) The address $\mathrm{d}-\mathbf{1}$ has a truth value and is read as 'there is/was a cat'. Existentially quantified addresses are open addresses.

An open address is closed when denoted in a subsequent clause by a definite term. Address closure, symbolized by a double slash, changes the address head from being a function to truth-values to being a reference function selecting an object (the referent) from a set of objects. Reference functions over individuals are typed $((\mathrm{e}, \mathrm{t}), \mathrm{e})$ (taking a set and delivering an individual), and are thus type-reducing. The reference function has been a source of discomfort to modern semantics because it cannot be defined within the confines of standard compositional model theory: there is no way of selecting one individual from a plural set by mathematical means. For it to work, an external input from cognition is needed - a further indication that meaning is subservient to cognition.

Let $\mathbf{D}$ contain $\mathbf{d} \mathbf{- 1}$ as above and also an open address d-4 $[\mathbf{a} \mid \operatorname{Mouse}(\mathrm{a})]$. Then a sentence like (5a), with the SA $(5 \mathrm{~b})$, results in the two parallel increments $(5 \mathrm{c})$ and $(5 \mathrm{~d})$.

(5a) The cat caught the mouse.

(5b)

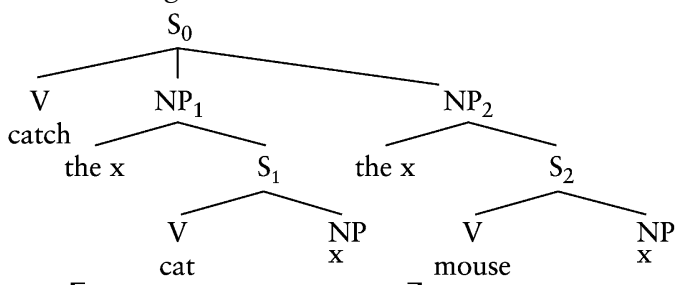

(5c) d-1 $[\mathrm{a} \mid \operatorname{Cat}(\mathrm{a}) / / \operatorname{Catch}(\mathbf{1 , 4})]$

(5d) d-4 $[\mathrm{a} \mid \operatorname{Mouse}(\mathrm{a}) / / \operatorname{Catch}(\mathbf{1 , 4})]$

$\mathrm{NP}_{1}$ in $(5 \mathrm{~b})$ reads 'the $x$ such that $x$ is a cat,' and analogously for $\mathrm{NP}_{2}$. In (5c) and (5d), the head a has been retyped from $((e, t), t)$ to $((e, t), e)$; the propositional function preceding closure - Cat(a) or Mouse(a) denotes the input set typed $(e, t)$. Catch $(1,4)$ is the proposition saying that the individual selected by the reference function $\boldsymbol{a}$ in $\mathbf{d}-\mathbf{1}$ - the cat - caught the individual selected in $\mathrm{d}-4$ - the mouse. IC first scans the predicate of $\mathrm{S}_{0}$. Catch being a binary lexical verb, IC is put to work on the definite $\mathrm{NP}_{1}$ first, to be followed by the definite $\mathrm{NP}_{2}$.

The denotation procedure $d$ for definite NPs is as follows: 
For any $\mathrm{NP}_{\mathrm{i}}$ under a definite-NP operator the:

- the takes the predicate of the $\mathrm{S}$ under $\mathrm{NP}_{\mathrm{i}}$ and selects the matching address $\mathbf{d}-\mathbf{n}$. There must, in principle, be only one such address in $\mathrm{D}$.

- $\mathbf{d}-\mathbf{n}$ is closed (if still open), and the SA-tree is added to the closed address, with the number $\boldsymbol{n}$ of $\mathbf{d}-\mathbf{n}$ for the $\mathrm{NP}_{\mathrm{i}}$-constituent.

Thus, $\mathrm{d}\left(\mathrm{NP}_{1}\right)$ in $(5 \mathrm{~b})$ selects $\mathbf{d}-\mathbf{1}[\mathrm{a} \mid \operatorname{Cat}(\mathrm{a})]$, and $\mathrm{NP}_{1}$ is replaced with $\mathbf{1} ; \mathbf{d} \mathbf{- 1}$ is closed and the SA-tree, with $\mathbf{1}$ in place, is added to the now closed $\mathbf{d} \mathbf{- 1}$ :

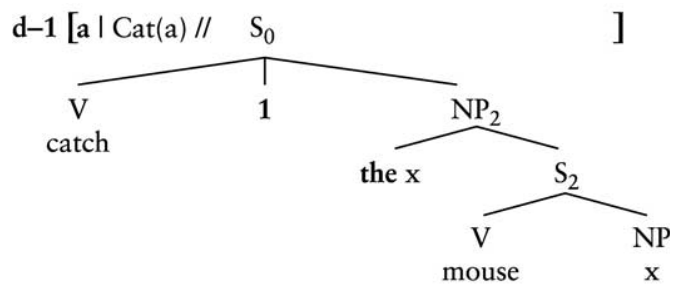

The procedure is repeated for $\mathrm{NP}_{2}$ in $\mathbf{d}-\mathbf{1}$, yielding the two parallel increments:

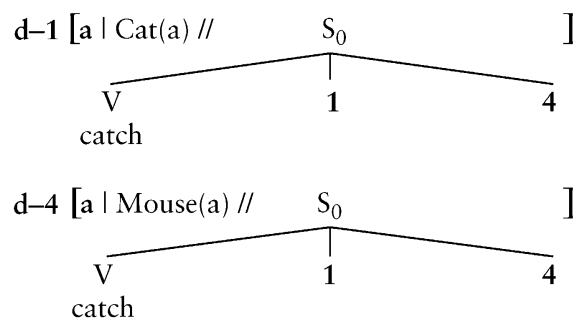

For practical reasons, trees are written as bracketed strings, giving $(5 \mathrm{c})$ and $(5 \mathrm{~d})$, respectively.

A sentence like (6a), with SA (6b) is incremented as follows, with D containing d-1 $[\mathbf{a} \mid \operatorname{Cat}(\mathrm{a})]$ :

(6a) The cat caught a mouse.

(6b)

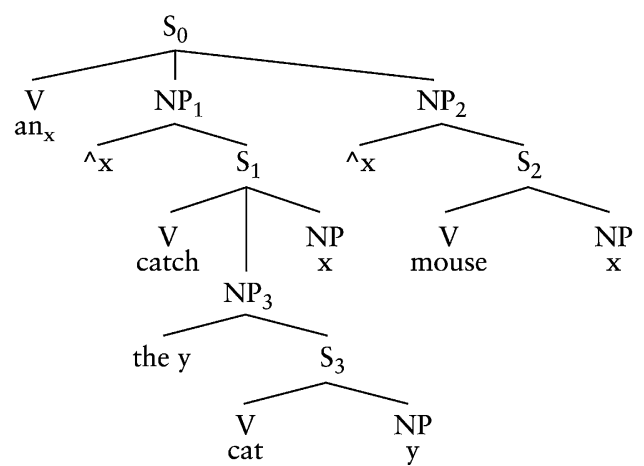

(6c) d-4 $[a \mid \operatorname{Mouse}(a), \operatorname{Catch}(1, a)]$

(6d) d-1 $[\mathrm{a} \mid \operatorname{Cat}(\mathrm{a}) / /[\mathrm{b} \mid \operatorname{Mouse}(\mathrm{b}), \operatorname{Catch}(\mathbf{1}, \mathrm{b})]]$

The new $\mathrm{d}-4$, created in virtue of $\mathrm{v}[\mathrm{an}]$, is fitted out with $S_{2}$ and $S_{1}$, in that order (with $a$ for $x$ ):

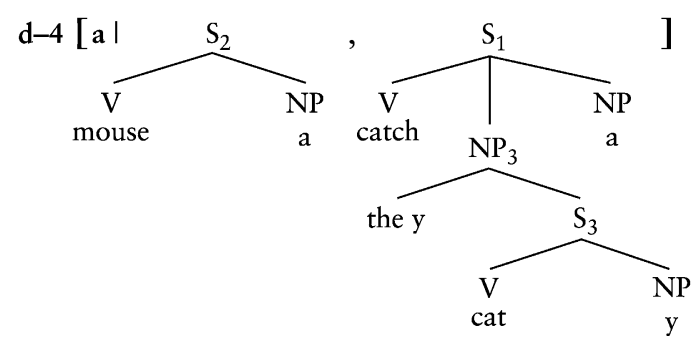

d-1 in (6d) contains a subordinate open address. An open address can be stored under another address $\mathbf{d}-\mathbf{n}$ provided it contains either the variable bound by $\mathbf{d}-\mathbf{n}$ (for open addresses) or the definite term $\mathbf{n}$ (for addresses after closure).

Double existential quantification is treated analogously. (7a), with SA ( $7 b$ ) yields the open address $(7 c)$ :

(7a) A cat caught a mouse.

(7b)

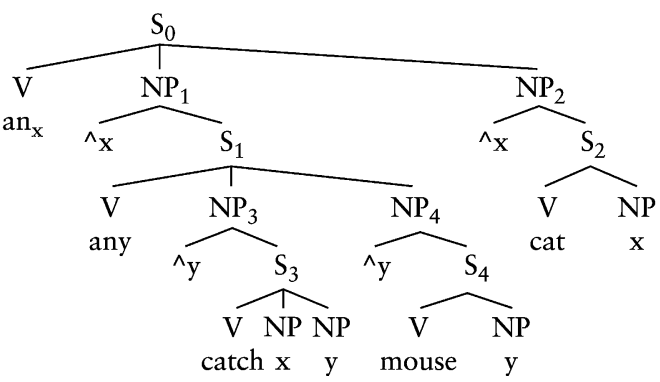

(7c) d-1 [a $\mid \operatorname{Cat}(a),[b \mid \operatorname{Mouse}(b), \operatorname{Catch}(a, b)]]$

IC causes $\mathbf{d}-\mathbf{1}$ to be set up in such a way that the cat-address takes scope over the mouse-address: 'there is a cat $a$ such that there is a mouse $b$ such that $a$ caught $b$ '. Since, however, it is possible to refer subsequently to the mouse caught by the cat, an independent open address for the mouse in question is also required. To that end, an address of the form (8) is set up in virtue of a process of inferential bridging:

(8) d-4 $[a \mid \operatorname{Mouse}(a),[b \mid \operatorname{Cat}(b), \operatorname{Catch}(b, a)]]$

Here the mouse-address takes scope over the cataddress, but the difference is irrelevant, as the simple existential quantifier is symmetrical. The discourse may now continue with definite terms like the cat that caught a mouse, closing $\mathrm{d}-1$, or the mouse that was caught by a cat, closing $\mathbf{d}-4$.

Now to plurality, which requires the notion of plural power set $\left(\mathbf{P}_{\mathrm{pl}}\right)$. For any set $X, \mathrm{P}_{\mathrm{pl}}(\mathrm{X})=\mathrm{P}(\mathrm{X})$ minus $\varnothing$ and all singleton sets. Thus, if $X$ has cardinality $n, \mathbf{P}_{\mathrm{pl}}(\mathrm{X})$ has cardinality $2^{\mathrm{n}}-(\mathrm{n}+1)$. Moreover, to distinguish distributive from group readings, the type-raising distributive operator ' $::$ ', defined over predicates, is required for the language of SAs. 
Let $[[\mathrm{P}(\mathrm{x})]]$ denote the extension of $\mathrm{P}(\mathrm{x})$ - the set of individuals $x$ such that $x$ satisfies $\mathrm{P}$ - then $[[\because: \mathrm{P}(\overline{\mathrm{x}})]]$ is defined as follows ( $\overline{\mathrm{x}}$ ranges over sets of individuals):

$$
[[\because: \mathrm{P}(\overline{\mathrm{x}})]]={ }_{\text {Def }} \mathbf{P}_{\mathrm{pl}}([[\mathrm{P}(\mathrm{x})]])
$$

The extension of $:: \mathrm{P}(\overline{\mathrm{x}})$ is thus the set of sets of at least two individuals $x$ such that each $x$ satisfies $P$; '::happy(the children)' reads 'the set of children in question is among the sets of at least two individuals each of whom is happy'. When $P$ is transitive and both of its terms are definite and plural, :: distributes indiscriminately over the subject and the object term referents. In the group reading, The men carried the bags reads as saying that the men as a group carried the bags as a group (while, say, the women carried the pots), leaving it open whether there were subgroups of men carrying subgroups of bags. The distributive reading says that each of the men carried one or more bags and each of the bags was carried by one or more men. The linguistic expression thus underdetermines the actual state of affairs.

An open plural address is normally of the form (9c), representing $(9 a)$ with SA $(9 b)$ :

(9a) There were cats.

(9b)

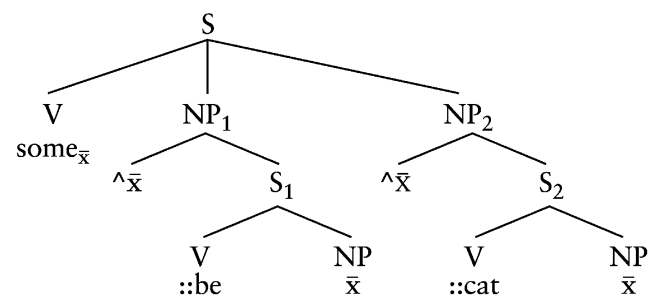

(9c) $\mathbf{d}-5[\overline{\mathbf{a}} \mid:: \operatorname{Cat}(\overline{\mathrm{a}})]$

Some is the plural existential quantifier again yielding truth just in case there is a nonnull intersection of at least one plural set of individuals of the two term extensions concerned, which now are sets of sets of individuals. Some is again an instruction to set up a new address of the right type. In (9c), $\overline{\mathbf{a}}$ represents plural SOME and binds the variable. Example (9c) thus requires that there be at least one set of at least two actually existing cats.

When Pred is second order by nature, typed $((e, t), t)$, the distributive operator :: is not needed. Then IC gives Pred $(\overline{\mathrm{a}})$, with the variable $\bar{a}$, as in the singular address (10) with the second-order predicate platoon (army unit consisting of men), representing There is/was a platoon:

$$
\text { (10) d-6 [ }[\bar{a} \mid \operatorname{Platoon}(\overline{\mathrm{a}})]
$$

The two forms can be combined, as in: (11a) d-5 $[\overline{\mathbf{a}} \mid::$ Cat $(\overline{\mathrm{a}})$, Disperse $(\overline{\mathrm{a}})]$

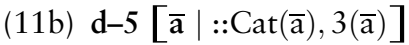

representing, respectively, Some cats dispersed and There were three cats. Mathematically, an address can be of any order, yet language stops at secondorder predicates requiring third-order addresses for their plurals, as in (12), which reads There are/ were platoons. Any higher-order nouns are treated as second-order.

(12) $\mathbf{d}-7[\overline{\mathbf{a}} \mid:: \operatorname{Platoon}(\overline{\mathrm{a}})]$

Plurals are difficult, and only some of the problems can be dealt with here. One such problem is the distinction between distributive and group readings. Group readings are incremented analogously to singular increments. Thus, given an open plural cataddress, such as $\mathrm{d}-5$ in (9c), the addition They ran away (group reading) is incremented as in (13a), where closure has turned $\overline{\mathbf{a}}$ into a reference function $(((e, t), t),(e, t))$, selecting a set from a set of sets:

(13a) d-5 [ $\overline{\mathbf{a}} \mid:: \operatorname{Cat}(\overline{\mathbf{a}}) / / \operatorname{Runaway}(5)]$

(13b) d-5 $[\overline{\mathbf{a}} \mid:: \operatorname{Cat}(\overline{\mathrm{a}}) / /:: \operatorname{Runaway}(5)]$

To compute the truth value of 'Run away(5),' it is necessary to type-raise the predicate run away from $(e, t)$ to $((e, t), t)$, so that it can process an input of type $(\mathrm{e}, \mathrm{t})$. Type-raising of a predicate $P$ implies that, despite the transition from individuals to groups, the satisfaction conditions of $P$ remain unchanged. This condition cannot be fulfilled by all predicates. Most nominal predicates, such as cat, dog, tree, house, being reserved for individual predication, disallow type-raising. Example (13a) is thus read intuitively as 'the set of cats referred to by $[\overline{\mathbf{a}} \mid:: \operatorname{Cat}(\overline{\mathbf{a}})]$ is a set of at least two individuals running away as a group'. The distributive reading of They ran away is incremented as in (13b), which lets the cats in question run away individually.

Typical group readings are found in sentences like:

(14a) The mice have been at the cheese.

(14b) The Americans were the first to land on the moon.

In their common reading, these do not imply that all the mice have been at the cheese, or that all Americans were the first to land on the moon, as they are about the mice, or the Americans, as a group.

Now consider (15a) with the SAs (15b) and (15c), each of which represents a group reading and a distributive reading. 
(15a) The men carried a bag.

(15b)

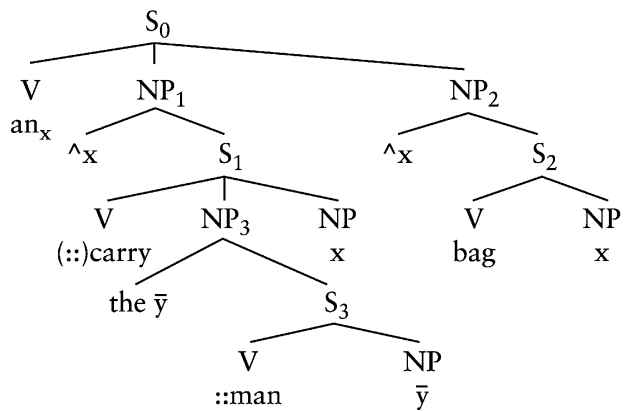

$(15 \mathrm{c})$

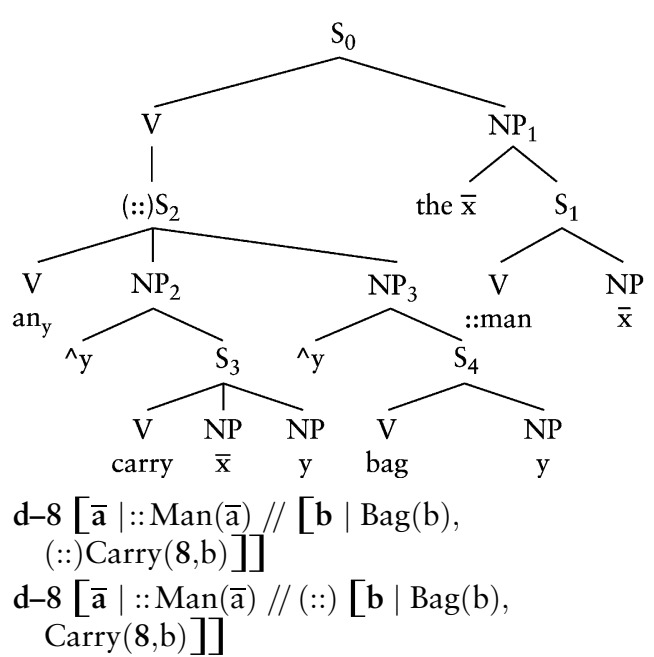

In $(15 \mathrm{c})$, the main predicate is the propositional function $S_{2}$, denoting the set of sets of at least two individuals that have a bag to carry, either as a group or individually. $S_{2}$ is the tree-structure version of what is known in logic as a lambda predicate. Like any other semantically appropriate predicate, $S_{2}$ can be placed under the distributive operator.

The four readings of $(15 \mathrm{a})$ are as follows $\left(\left[16 \mathrm{~b}_{2}\right]\right.$ and $\left[16 c_{2}\right]$ are equivalent):

$\left(16 b_{1}\right)$ 'there was one bag carried by each of the men individually'

$\left(16 b_{2}\right)$ 'there was one bag collectively carried by the men'

$\left(16 c_{1}\right)$ 'each of the men carried a bag'

$\left(16 c_{2}\right)$ 'the group of men collectively carried a bag'

Let $\mathbf{D}$ contain an open address $\mathbf{d}-8[\overline{\mathbf{a}} \mid:: \operatorname{Man}(\overline{\mathbf{a}})]$. Then for (15b) IC creates a new open address d-9 [b | $\operatorname{Bag}(\mathrm{b}),(::) \operatorname{Carry}(\mathbf{8}, \mathrm{b})]$, saying that there is/ was a bag which the men of $\mathrm{d}-\mathbf{8}$ carried collectively (without ::) or individually (with ::). $\mathbf{d}-\mathbf{8}$ is now closed, analogously to $d-\mathbf{1}$ in $(6 d)$, representing the two readings $\left(16 b_{1}\right)$ and $\left(16 b_{2}\right)$. This gives $(15 d)$. Analogously for (15e). The group readings of (15b) and (15c) result in an identical d-8. Inferential bridging sets up an open singular bag-address in the group reading of (15e) - that is, $\left(16 c_{2}\right)$ - but the distributive $\left(16 c_{1}\right)$ requires an open plural bag-address, enabling subsequent reference to the bags that the men carried. The formal treatment of this form of inferential bridging has not been elaborated so far.

The closure operation is important for empirical reasons, as appears, for example, from (17a) and (17b), whose semantic distinction is unaccounted for in most semantic theories:

(17a) Nob sent few letters that were rude.

(17b) Nob sent few letters, and they were rude.

The difference corresponds with the closure operation. Let $\mathrm{D}$ already contain an address $\mathbf{d - 1 0}[\mathrm{a} \mid$ 'Nob' (a)]. Both (17a) and (17b) set up a new address $\mathbf{d} \mathbf{- 1 1}$ for the letters Nob sent. For (17a), d-11 is left open, but for (17b), d-11 is closed:

$$
\begin{aligned}
& \text { (18a) d-11 }[\overline{\mathbf{a}} \mid:: \operatorname{Letter}(\overline{\mathbf{a}}),:: \operatorname{Rude}(\overline{\mathbf{a}}),:: \operatorname{Send}(\mathbf{1 0}, \overline{\mathbf{a}}), \\
& \text { Few }(\overline{\mathbf{a}})] \\
& \text { (18b) d-11 }[\overline{\mathbf{a}} \mid:: \operatorname{Letter}(\overline{\mathbf{a}}),:: \operatorname{Send}(\mathbf{1 0}, \overline{\mathbf{a}}), \operatorname{Few}(\overline{\mathbf{a}}) / / \\
& :: \operatorname{Rude}(\mathbf{1 1})]
\end{aligned}
$$

We now consider the universal quantifier all, as in (19a) with SA (19b) and the IC-result (19c) treated as a binary higher-order predicate. $\mathrm{D}$ already contains d-12 $[\overline{\mathbf{a}} \mid::$ Mouse $(\overline{\mathrm{a}})]$ ('there are mice'):

(19a) All (the) mice escaped.

(19b)

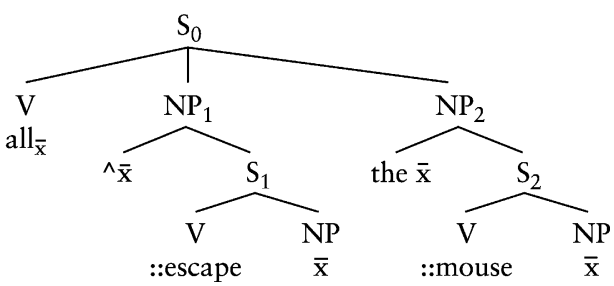

(19c) d-12 [ $\overline{\mathbf{a}} \mid:: \operatorname{Mouse}(\overline{\mathrm{a}}) / /$ All $([:: \operatorname{Escape}(\overline{\mathrm{x}})]$, (12))]

All takes a first-order definite plural object term and a second-order set-denoting subject term, delivering truth just in case the object set is an element of the subject set. This analysis provides a unified solution to the type problem caused by standard analyses for sentences like All (the) mice dispersed. The analysis $\forall \mathrm{x}$ (mouse $(\mathrm{x}) \rightarrow$ disperse $(\mathrm{x}))$ or, in generalized quantification, $\forall \mathrm{x}(\operatorname{disperse}(\mathrm{x})$, mouse $(\mathrm{x}))$ will not do as 'disperse $(\mathrm{x})$ ' is of the wrong type. $\mathbf{d - 1 2}$ is closed, following the definite reference to a particular set of mice. '[::Escape $(\overline{\mathrm{x}})]$ ' denotes the set of sets of at least two individually escaping individuals. 'All([::Escape $(\bar{x})],(12)$ )' says that the mice in question form a set of at least two individually escaping individuals.

This makes $\operatorname{All}([\because: \operatorname{Escape}(\overline{\mathrm{x}})],(\mathbf{1 2}))$ equivalent to ::Escape(12), and it gives all existential import, as in traditional predicate calculus. The difference between 
all and plural the seems to be that while they both select the set $M$ of elements defined by the address head after closure (the mice), and let the sentence say that $M$ is a member of the set of sets $E$ defined by ::escape, all specifically requires (redundantly) that no member of $M$ be left out. This makes all unsuitable for higher order predicates like numerous, but suitable for other higher-order predicates like disperse or sit in a circle. Every distinguishes itself from all mainly in that every excludes group readings. The specifics of each are not touched upon here.

Finally, consider (20), which has both a group and a distributive reading. The existential predicates few and many say that the intersecting set required by the existential quantifier is small or large, respectively:

(20a) Few cats caught many mice.

(20b)

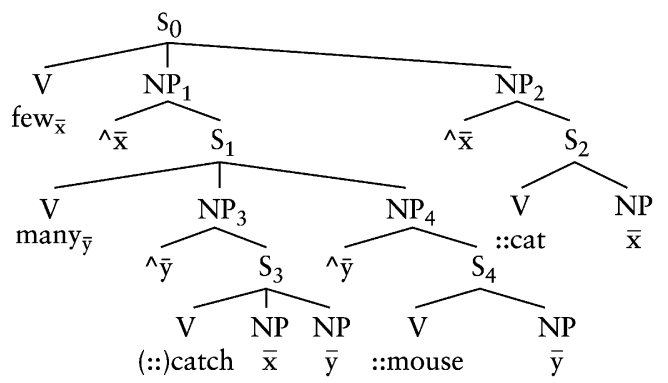

(20c) d-7 $[\overline{\mathbf{a}} \mid:: \operatorname{Cat}(\mathrm{a}), \operatorname{Few}(\overline{\mathrm{a}}),[\overline{\mathrm{b}} \mid:: \operatorname{Mouse}(\overline{\mathrm{b}})$, $\operatorname{Many}(\bar{b}),(::) \operatorname{Catch}(\overline{\mathrm{a}}, \overline{\mathrm{b}})]]$

The group reading is unproblematic: 'a small group of cats caught a large group of mice'. In this reading subsequent definite reference can be made to the large group of mice, as in These mice had escaped from a laboratory, which requires the inferentially added address:

(21) d-12 $[\overline{\mathbf{a}} \mid:: \operatorname{Mouse}(\overline{\mathrm{a}}), \operatorname{Many}(\overline{\mathrm{a}}),[\overline{\mathrm{b}} \mid:: \operatorname{Cat}(\overline{\mathrm{b}})$, $\operatorname{Few}(\bar{b}), \operatorname{Catch}(\bar{b}, \bar{a})]]$

But in the distributive reading, with ::Catch $(\bar{b}, \bar{a})$, subsequent definite reference is not possible, which means that inferential bridging of the kind at issue must be blocked. The passive of (20a):

(22) Many mice were caught by few cats.

is equivalent to (20a) only in the group reading. In the distributive reading, scope differences destroy the equivalence. As with the distributive reading of (15e), the formal criterion for this blocking has as yet not been elaborated.

Negation is treated as an abstract predicate in SAstructure, like the quantifiers. Its subject-S is what is normally called its scope. Thus, (23a) has the SA (23b): (23a) The mouse did not escape.

(23b)

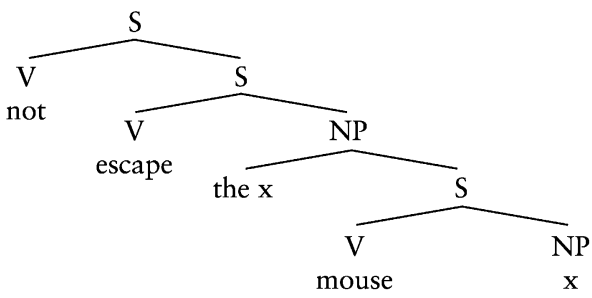

(23c) d-4 [a | Mouse(a) // *Escape(4)]

For IC, negation is an instruction preventing the addition of the predication immediately following it to the address in question. The non-negated predication must be normally incrementable ('have the right papers'). IC takes the subject-S of not in the SA-tree and processes it first without negation. Subsequently, not places an asterisk before the predicate. In the case at hand, there must, therefore, be an appropriate mouse-address available in $\mathrm{D}$. * Is presuppositionpreserving and differs therefore from the negation in standard logic (see Presupposition).

Open addresses can be negated, as in (24a), incremented as (24b):

(24a) No mouse was caught.

(24b) d-n [*a $\mid$ Mouse(a), Be caught(a)]

This blocks the addition of 'a | Mouse(a), Be caught(a)' to any address in D. Therefore, negated open addresses cannot be closed: there is nothing to close (but see [27] below).

Example (24a) is not equivalent with (25a) and (25b) but these are equivalent with (26a) and (26b).

(25a) All the mice were not caught.

(25b) d-12 [ $\overline{\mathbf{a}} \mid:: \operatorname{Mouse}(\overline{\mathbf{a}}) / /$ All $([*:: \operatorname{Be~caught}(\overline{\mathbf{x}})]$, $(12))]$

(26a) None of the mice were caught.

(26b) d-12 $[\overline{\mathbf{a}} \mid::$ Mouse $(\overline{\mathrm{a}}) / /[* \mathrm{~b} \mid \in \mathbf{1 2}(\mathrm{b})$, Be caught $(b)]]$

\section{Subdomain Structures}

A D may contain subdomains, which are either alternatives or subordinates. Alternative subdomains are created by or (disjunction) and if (implication). $\mathrm{i}(\mathrm{A}$ or $\mathrm{B})$ is ' $\mathrm{A} / \operatorname{not}(\mathrm{A})$ and $\mathrm{B}$ ' - with the alternative disjuncts ' $\mathrm{A}$ ' and ' $\operatorname{not}(\mathrm{A})$ and $\mathrm{B}$ '. The truth condition is that at least one disjunct be true. The tacit exclusion (negation) of the first disjunct in the second rests on the True Alternatives Condition for Ds, requiring that the alternative increments under disjunction be truly distinct. This explains the much debated 
'exclusive' character of or as resulting from principles of coherent discourse construction.

Implication is like disjunction: $\mathrm{i}$ (if $\mathrm{A}$ then $\mathrm{B}$ ) consists of two alternative increments: 'A and B / not $(\mathrm{A})$ ', with the tacit inference that when ' $\operatorname{not}(\mathrm{A})$ ' is chosen, ' $\mathrm{B}$ ' is excluded, since if ' $\mathrm{B}$ ' is added after 'not(A)', the disjunction of 'A' and 'not(A)' has been vacuous.

This analysis accounts for definite anaphora after a negative existential disjunct, as in (27a), or a positive existential antecedent clause, as in (27b):

(27a) Either Nancy has no husband or he is Norwegian.

(27b) If Nancy has a husband, he is Norwegian.

Example (27a) is explained by the tacit incrementation of 'she has a husband' in the second disjunct, and (27b) by the fact that $\mathrm{i}$ (he is Norwegian) is made possible by the preceding i(Nancy has a husband) in the same alternative.

Subordinate subdomains are a special kind of address. Like ordinary addresses, they can be open or closed. They also have D-properties, in that they may contain their own addresses, increments, and instructions. They are represented both as a special kind of address and as an indexed domain. A sentence like (28a) is incremented as (28b), where the variable $D$ ranges over virtual facts. $\left(28 b_{1}\right)$ reads 'there is a possible fact D'. Possible carries an instruction to set up a subdomain specifying the virtual fact in question. This subdomain is represented in $\left(28 b_{2}\right)$ read as 'there is a planet called "Minerva" and it is inhabited':

(28a) There may be a planet Minerva and it may be inhabited.

$$
\begin{gathered}
\left(28 b_{1}\right) \text { D-1 }[\mathrm{D} \mid \operatorname{Possible}(\mathrm{D})] \\
\left(28 \mathrm{~b}_{2}\right) \mid \rightarrow \text { D-1: d-1 }[\mathrm{a} \mid \text { "Minerva"(a), Planet(a) // } \\
\text { Inhabited }(\mathbf{1})]
\end{gathered}
$$

It in (28a) refers opaquely, as it finds its antecedent within D-1. Transparent reference, with $\mathbf{d}-\mathbf{1 3}$ given in the superordinate $\mathbf{D}$, is shown in (29). In (29c), d-13 is closed and the predication D-1[Inhabited(13)] is added, saying that $\mathbf{1 3}$ is represented as being inhabited in subdomain $\mathbf{D}-\mathbf{1}$ :

(29a) There is a planet Minerva and it may be inhabited.

(29b) D-1 [D | Possible(D)]

D-1: Inhabited(13)

(29c) d-13 [ a | "Minerva" (a), Planet(a) // D-1 [Inhabited(13)]]

A few general principles hold for subdomains. First, addresses from the commitment domain 'percolate downward' into subdomains. As shown in (29), where $\mathbf{d} \mathbf{- 1 3}$ in $\mathbf{D}-\mathbf{1}$ is taken from $\mathbf{D}$. This downward percolation is stopped only if the subdomain in question explicitly blocks the address in question. Then, presuppositions of clauses incremented in subdomains 'percolate upward' into higher domains, including $\mathrm{D}$, unless blocked either by their explicit negation or by lack of cognitive backing. This process is called "PROJECTION. Both processes follow from the principles of Maximal Unity (MaU) and Minimal Size (MiS), which serve the functional purpose of ensuring maximal unity and coherence in the overall $\mathrm{D}$-structure.

Anaphora may delve into subdomains under intensional predicates. In (30), for example, the brother Marion believes she has is anaphorically referred to by he under the intensional predicate be the talk of the town:

(30) Marion believes that she has a brother, and he is the talk of the town.

The machinery of the incremental construction of discourse domains and subdomains is the main explanatory factor for the lack of substitutivity salva veritate in intensional contexts, which has been the dominant driving force in theoretical semantics.

See also: Anaphora: Philosophical Aspects; Extensionality and Intensionality; Negation; Presupposition; Virtual Objects.

\section{Bibliography}

Fauconnier G (1985). Mental spaces. Aspects of meaning construction in natural language. Cambridge: MIT Press.

Gazdar G (1979). Pragmatics. Implicature, presupposition and logical form. New York, San Francisco, London: Academic Press.

Geach P (1962). Reference and generality. An examination of some medieval and modern theories. Ithaca NY: Cornell University Press.

Isard S (1975). 'Changing the context.' In Keenan E (ed.) Formal semantics of natural language. Cambridge: Cambridge University Press. 287-296.

Seuren P A M (1972). 'Taaluniversalia in de transformationele grammatika.' Leuvense Bijdragen 61, 311-370.

Seuren P A M (1975). Tussen taal en denken. Een empirische bijdrage tot de semantiek. Utrecht: Oosthoek, Scheltema, Holkema.

Seuren P A M (1985). Discourse semantics. Oxford: Blackwell.

Seuren P A M (1996). Semantic syntax. Oxford: Blackwell. 